\title{
Interaction of whispering gallery modes in integrated optical micro-ring or -disk circuits: Hybrid CMT model
}

\author{
Ellen F. Franchimon ${ }^{1}$, Kirankumar R. Hiremath ${ }^{2}$, Remco Stoffer ${ }^{3}$, Manfred Hammer ${ }^{1}$ * \\ ${ }^{1}$ University of Twente, Enschede, The Netherlands \\ ${ }^{2}$ Indian Institute of Technology, Jodhpur, India \\ ${ }^{3}$ PhoeniX Software, Enschede, The Netherlands
}

\begin{abstract}
Whispering gallery modes supported by open circular dielectric cavities are embedded into a non-parametric 2-D frequency domain hybrid coupled mode theory framework. Regular aggregates of these cavities, including straight access channels, are investigated. The model enables convenient studies of the guided-wave scattering process, the response of the circuit to guided wave excitation. Transmission resonances can be characterized directly in terms of resonance frequency and linewidth by computing supermodes of the entire composite circuits, comprising both cavities and bus waveguides. Examples of single ring- and disk-filters, a coupledresonator optical waveguide, and a three-cavity photonic molecule in a reflector configuration allow the approach to be assessed.
\end{abstract}

Keywords: Optical micro-ring and micro-disk resonators, whispering gallery resonances, numerical / analytical modeling, coupled mode theory.

PACS codes: $42.82 .-\mathrm{m}$ 42.82. Bq 42.82.Et

\section{Introduction}

Circuits made of photonic micro-resonators [1, 2, 3] have already for more than a decade been intensely investigated, typically with a view to applications in e.g. optical telecommunication or optical sensing. Our focus is on coupled circular dielectric ring- or disk-cavities. When it comes to modelling and design, but also to understanding, techniques relying on coupled mode theory (CMT) offer frequently a more practical alternative to usually computationally quite expensive rigorous numerical methods. As the present work continues our preceding study [4], we refer to that article and the citations therein for a brief overview of techniques that are relevant in the present context.

Circular micro-resonators are traditionally described in terms of a parametric model [5, 6]. Bend modes supported by curved waveguides or curved dielectric interfaces [7] represent the optical field in the cavity. For given real excitation frequency these modes are characterized by a complex angular propagation constant. Coupling regions are defined where the cavity(-ies) and bus waveguides are close. The mode amplitudes (functions) are expected to change while traversing these regions, while outside the fields are assumed to propagate independently. Resonances appear as extrema in the transmission characteristics when scanning over excitation frequency, i.e. must be attributed to the entire composite device.

There is, however, a dual, equally justified viewpoint [8], that first considers the separate optical cavities, here the separate dielectric rings or disks. Their "native" resonances, known as Whispering Gallery Modes (WGMs), are characterized by a complex eigenfrequency and integer angular wavenumber [9] (for lack of a better term, we apply the name WGM also to cavities with more than one circular interface). If the cavities are brought together and / or close to a bus waveguide, one expects the WGMs to interact, where "interaction" now means adjustment of their individual coefficients. Building on the preliminary results of [10], it is our aim here to realize this second viewpoint by introducing WGMs into a framework of hybrid analytical / numerical coupled mode theory (HCMT, [11]). The previous bend-mode view has been explored, in an HCMT context, in Ref. [4]. We shall see below that both approaches lead to close results, at least for the present set of parameters.

For a configuration without excitation, looking for nontrivial solutions establishes an eigenvalue problem for the "supermodes" of the circuit. On the one hand, when applied e.g. to a cluster of cavities ("atoms") that constitute a photonic "molecule", this permits one to investigate the lift of degeneracies of the atoms' eigenfrequencies

* $\mathrm{MESA}^{+}$Institute for Nanotechnology, University of Twente

Phone: $+31 / 53 / 489-3448$
Fax: $+31 / 53 / 489-3996$

P.O. Box 217, 7500 AE Enschede, The Netherlands E-mail:m.hammer@utwente.nl 
due to the presence of the neighboring atoms. On the other hand, the supermode reasoning can be applied to composite circuits that comprise cavities and bus channels, with the fields in the waveguides restricted to outgoing waves. Frequencies and linewidths of (transmission) resonances are thus computed directly, i.e. without having to carry out frequency scans. Here one might observe similarities to the treatment of resonant 1-D multilayer structures in terms of quasi-normal modes (QNMs, [12, 13]). We do not, however, attempt any rigorous expansion into a complex frequency basis of the composite problem. Rather we will reason, at least qualitatively, along the procedure outlined in [14, 15, 16], where resonances of the transmission problem are approximated by combining (supermode) resonances of individual and composite cavities with suitable expressions for the incoming waves.

In particular, the WGM-HCMT supermode approach permits one to conveniently investigate coupling induced shifts of resonance frequencies [17] and related effects. The issue attracted further recent interest; examples are the parametric frequency- and time-domain models of Refs. [18, 19], also combined with numerical analysis [20] and experimental observations [21]. Contrary to respective statements in Ref. [17], these coupling-induced phase shifts can well be predicted by CMT, at least by the (ab-initio) variant discussed here.

The properties of WGMs, for the parameters that we consider in this paper, are briefly discussed in Section 2 Section 3 outlines the present coupled mode framework, and introduces the notions on supermode and perturbation analysis. The examples in Section 4 provide benchmarks, and illustrate different aspects of the previous theoretical approach.

\section{Whispering gallery modes of micro-rings and -disks}

WGMs of circular cavities serve here as prototypes of the eigenmodes of open dielectric cavities. Adhering to the analytical 2-D model of [9], using an ansatz of separable fields in polar coordinates, the relevant scalar 2-D Helmholtz equation transforms into an eigenvalue problem of Bessel type for the radial function, with piecewise constant coefficients. Piecewise solutions are sought in terms of Bessel and Hankel functions. The choice of the Hankel function of second kind in the external region, for a time dependence $\sim \exp \left(\mathrm{i} \omega^{\mathrm{c}} t\right)$ for eigenfrequencies $\omega^{\mathrm{c}}$ with positive real and imaginary parts, realizes the boundary condition of purely outgoing waves. Analytical solutions are found by tracking roots through a complex secant method. Our implementation relies on routines for complex Bessel and Hankel functions from [22, 23]. To generate initial root estimates, the related bend mode problems are translated to equivalent straight waveguides [24], followed by feeding restricted staircase approximations of the resulting effective refractive index profiles to the 1-D multilayer slab mode solver of [25]. Figure 1 summarizes the properties of some of the WGMs that will play a role in Section 4.
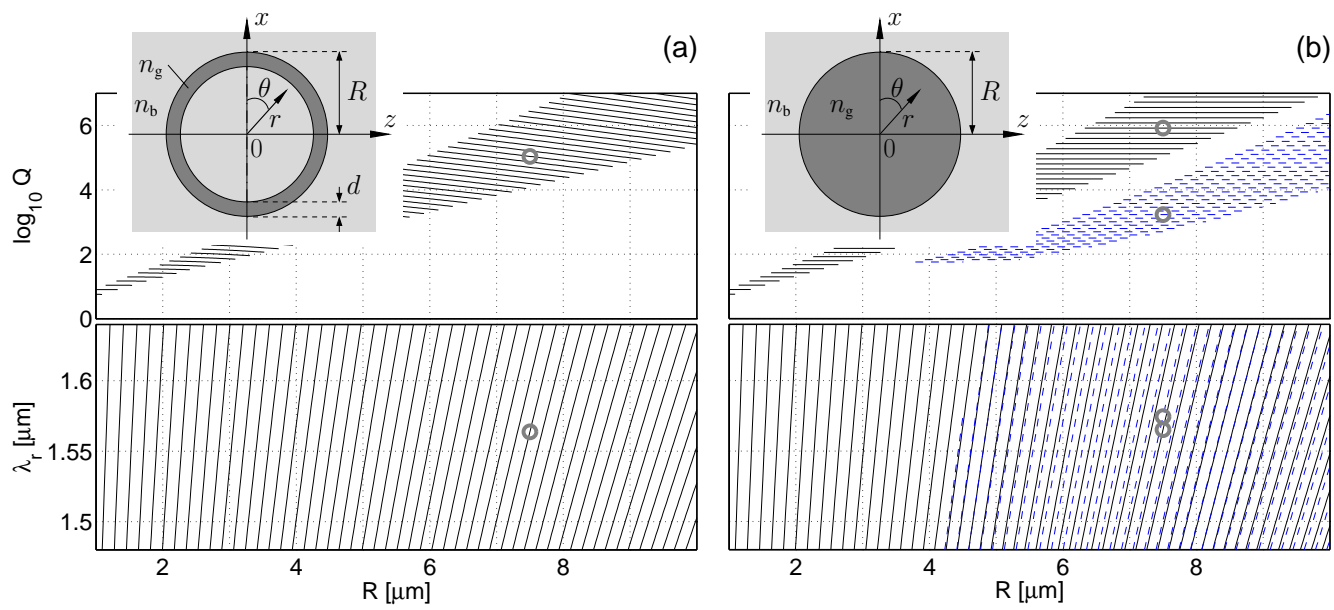

(b)

(c)

Figure 1: Resonance wavelengths $\lambda_{r}$ and quality factors $Q$ of WGMs supported by 2-D dielectric rings (a) and disks (b) with varying radii $R$ in a wavelength region around $1.55 \mu \mathrm{m}$; parameters as given for Figure 2. (c): profile of the ringWGM indicated by the marker in (a), for $R=7.5 \mu \mathrm{m}$; absolute value (top) and time snapshot (bottom) of the principal electric component $E_{y}$. See Figure $6(a, d)$ for the profiles of the disk-WGMs marked in (b).

We adopt a notation $\operatorname{WGM}(l, m)$ for the characterization of the WGMs in terms of two "quantum numbers", the number of radial minima $l$ in the principal electric field component of the mode profile, and the angular wavenumber $m$. Adhering to common notions, the eigenfrequencies $\omega^{\mathrm{c}}$ are specified in terms of the related 
resonance wavelength $\lambda_{\mathrm{r}}=2 \pi \mathrm{c} / \operatorname{Re} \omega^{\mathrm{c}}$, the Q-factor $Q=\operatorname{Re} \omega^{\mathrm{c}} /\left(2 \operatorname{Im} \omega^{\mathrm{c}}\right)$ associated with the resonance, and by the ratio $\Delta \lambda=\lambda_{\mathrm{r}} / Q$, which corresponds to the linewidth (full-width at half maximum) of the outgoing radiation [26]. A time animation of the physical waves shows the field rotating clockwise as one compound, with outwards escaping radiation, slowly decaying in time. All modes are twofold degenerate; fields WGM $(l,-m)$ corresponding to anticlockwise rotation and respectively escaping radiation constitute valid solutions of the WGM-eigenvalue problem as well.

\section{Hybrid analytical / numerical coupled mode theory}

The HCMT approach will be outlined for the single ring filter configuration as introduced in Figure 2, adapted from Ref. [4]. The parameters apply correspondingly also to all other configurations in this paper. Our primary interest is in the scattering problem, i.e. in determining the relative guided wave transmission $T$ and power drop $D$ for given excitation, here in the upper left port. Unidirectional propagation, as indicated by the arrows in Figure 2, will be considered first. Extension towards further channels or cavities, towards multimode elements, or towards bidirectional wave propagation should be straightforward [11].

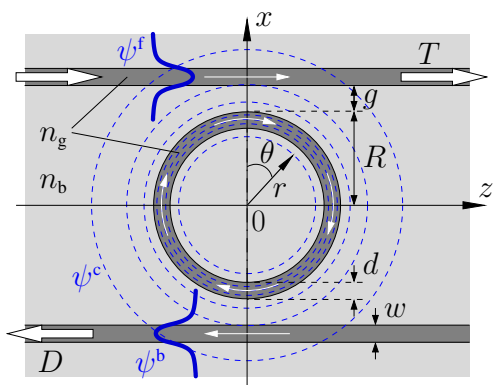

Figure 2: Filter device consisting of a cavity ring between two straight bus waveguides, a 2-D configuration described in Cartesian coordinates $x, z$ or polar coordinates $r, \theta$. Parameters: refractive indices $n_{g}=1.5$ (guiding regions), $n_{b}=1.0$ (background), cavity radius $R=7.5 \mu \mathrm{m}$, core width $d=0.75 \mu \mathrm{m}$, bus waveguides, core width $w=0.6 \mu \mathrm{m}$, gaps $g=0.3 \mu \mathrm{m}$. All simulations are restricted to 2-D, uniform for TE polarization, where the single principal electric field component $E_{y}$ is perpendicular to the $x$-z-plane of interest. We consider a spectral region around the target vacuum wavelength $\lambda \approx 1.55 \mu \mathrm{m}$.

\subsection{HCMT procedure}

We aim at approximate solutions, in 2-D, of the homogeneous frequency-domain Maxwell equations

$$
\boldsymbol{\nabla} \times \boldsymbol{H}-\mathrm{i} \omega \epsilon_{0} \epsilon \boldsymbol{E}=0, \quad-\boldsymbol{\nabla} \times \boldsymbol{E}-\mathrm{i} \omega \mu_{0} \boldsymbol{H}=0,
$$

for the optical electric and magnetic fields $\boldsymbol{E}, \boldsymbol{H}$. The stationary fields oscillate $\sim \exp (\mathrm{i} \omega t)$ in time with the (real) excitation frequency $\omega=2 \pi \mathrm{c} / \lambda$, specified by the excitation wavelength $\lambda$, for vacuum speed of light $\mathrm{c}$. The HCMT model relies on a plausible "template", an ansatz for the overall electromagnetic field, which, in case of the filter of Figure 2, reads

$$
\left(\begin{array}{c}
\boldsymbol{E} \\
\boldsymbol{H}
\end{array}\right)(x, z)=f(z) \boldsymbol{\psi}^{\mathrm{f}}(x, z)+b(z) \boldsymbol{\psi}^{\mathrm{b}}(x, z)+\sum_{j} c_{j} \boldsymbol{\psi}_{j}^{\mathrm{c}}(r, \theta),
$$

implying the relations $r(x, z), \theta(x, z)$ between polar and Cartesian coordinates. Here $\boldsymbol{\psi}^{\mathrm{f}, \mathrm{b}}(x, z)=$ $(\tilde{\boldsymbol{E}}, \tilde{\boldsymbol{H}})^{\mathrm{f}, \mathrm{b}}(x) \exp (\mp \mathbf{i} \beta z)$ are the forward/backward modes guided by the upper/lower bus channels at frequency $\omega$, with profiles $(\tilde{\boldsymbol{E}}, \tilde{\boldsymbol{H}})^{\mathrm{f}}$, b , propagation constants $\mp \beta$, and $z$-dependent amplitudes $f$ and $b$. A number (index $j$ ) of WGMs $\boldsymbol{\psi}_{j}^{\mathrm{c}}(r, \theta)=(\tilde{\boldsymbol{E}}, \tilde{\boldsymbol{H}})_{j}^{\mathrm{c}}(r) \exp \left(-\mathrm{i} m_{j} \theta\right)$ with mode profiles $(\tilde{\boldsymbol{E}}, \tilde{\boldsymbol{H}})_{j}^{\mathrm{c}}$ and integer angular wavenumbers $m_{j}$ enter with coefficients $c_{j}$. By discretizing the functions $f, b$ in terms of 1-D, here linear finite elements (FEs) [11], the template (2) can be given the abstract form

$$
\left(\begin{array}{c}
\boldsymbol{E} \\
\boldsymbol{H}
\end{array}\right)(x, z)=\sum_{k} a_{k}\left(\begin{array}{c}
\boldsymbol{E}_{k} \\
\boldsymbol{H}_{k}
\end{array}\right)(x, z)
$$

of a sum over "modal elements" $\left(\boldsymbol{E}_{k}, \boldsymbol{H}_{k}\right)$ which, in case of the bus channels, combine the mode profiles with the exponential dependences on the propagation coordinates and the familiar FE triangle functions. For the cavity, the WGM profiles appear as modal elements, without further factors. The set of coefficients $a_{k} \in$ $\left\{f_{j}, b_{j}, c_{j}\right\}$ includes correspondingly the discretized functions $f$ and $b$ as well as the WGM amplitudes $c_{j}$. Of 
these, the coefficients that relate to the incoming wave in the upper left port and to the zero excitation in the lower right port are given; all others need to be determined.

To do so, the relevant Maxwell equations (11) are multiplied by trial fields $\boldsymbol{F}, \boldsymbol{G}$ and integrated over a suitable (2-D) computational domain [11]. One arrives at a weak form of Eqs. (1), which, for reasons that become apparent in Section 3.2, is here written as

$$
\iint \mathcal{A}(\boldsymbol{F}, \boldsymbol{G} ; \boldsymbol{E}, \boldsymbol{H}) \mathrm{d} x \mathrm{~d} z-\omega \iint \mathcal{B}(\boldsymbol{F}, \boldsymbol{G} ; \boldsymbol{E}, \boldsymbol{H}) \mathrm{d} x \mathrm{~d} z=0 \text { for all } \boldsymbol{F}, \boldsymbol{G},
$$

where

$\mathcal{A}(\boldsymbol{F}, \boldsymbol{G} ; \boldsymbol{E}, \boldsymbol{H})=\boldsymbol{F}^{*} \cdot(\boldsymbol{\nabla} \times \boldsymbol{H})-\boldsymbol{G}^{*} \cdot(\boldsymbol{\nabla} \times \boldsymbol{E}), \quad \mathcal{B}(\boldsymbol{F}, \boldsymbol{G} ; \boldsymbol{E}, \boldsymbol{H})=\mathrm{i} \epsilon_{0} \epsilon \boldsymbol{F}^{*} \cdot \boldsymbol{E}+\mathrm{i} \mu_{0} \boldsymbol{G}^{*} \cdot \boldsymbol{H}$

Upon inserting the generalized template (3) for $\boldsymbol{E}, \boldsymbol{H}$, and restricting Eq. (4) to the set of modal elements $(\boldsymbol{F}, \boldsymbol{G}) \in\left\{\left(\boldsymbol{E}_{k}, \boldsymbol{H}_{k}\right)\right\}$, this Galerkin procedure leads to a system of linear equations of the form

$$
\sum_{k}\left(A_{l k}-\omega B_{l k}\right) a_{k}=0, \text { for all } l
$$

with "overlaps" of modal elements

$$
A_{l k}=\iint \mathcal{A}\left(\boldsymbol{E}_{l}, \boldsymbol{H}_{l} ; \boldsymbol{E}_{k}, \boldsymbol{H}_{k}\right) \mathrm{d} x \mathrm{~d} z, \quad B_{l k}=\iint \mathcal{B}\left(\boldsymbol{E}_{l}, \boldsymbol{H}_{l} ; \boldsymbol{E}_{k}, \boldsymbol{H}_{k}\right) \mathrm{d} x \mathrm{~d} z .
$$

One now groups the coefficients $\boldsymbol{a}=(\boldsymbol{u}, \boldsymbol{g})$ such that $\boldsymbol{u}$ represents the actual unknowns, while $\boldsymbol{g}$ corresponds to the given excitation, and arranges the system (6) accordingly:

$$
\begin{aligned}
& {\left[\left(\begin{array}{cc}
\mathrm{A}_{\mathrm{uu}} & \mathrm{A}_{\mathrm{ug}} \\
\mathrm{A}_{\mathrm{gu}} & \mathrm{A}_{\mathrm{gg}}
\end{array}\right)-\omega\left(\begin{array}{ll}
\mathrm{B}_{\mathrm{uu}} & \mathrm{B}_{\mathrm{ug}} \\
\mathrm{B}_{\mathrm{gu}} & \mathrm{B}_{\mathrm{gg}}
\end{array}\right)\right]\left(\begin{array}{c}
\boldsymbol{u} \\
\boldsymbol{g}
\end{array}\right)=0,} \\
& \text { or } \quad \mathrm{K}_{\mathrm{u}} \boldsymbol{u}=-\mathrm{K}_{\mathrm{g} g} \boldsymbol{\text { with }} \quad \mathrm{K}_{\mathrm{u}}=\left(\begin{array}{c}
\mathrm{A}_{\mathrm{uu}}-\omega \mathrm{B}_{\mathrm{uu}} \\
\mathrm{A}_{\mathrm{gu}}-\omega \mathrm{B}_{\mathrm{gu}}
\end{array}\right), \quad \mathrm{K}_{\mathrm{g}}=\left(\begin{array}{c}
\mathrm{A}_{\mathrm{ug}}-\omega \mathrm{B}_{\mathrm{ug}} \\
\mathrm{A}_{\mathrm{gg}}-\omega \mathrm{B}_{\mathrm{gg}}
\end{array}\right) .
\end{aligned}
$$

This last overdetermined system (9) can be handled in a least squares sense. One obtains, for given input $\boldsymbol{g}$, the response $\boldsymbol{u}$ at a prescribed excitation frequency $\omega$ as the solution of

$$
\mathrm{K}_{\mathrm{u}}^{\dagger} \mathrm{K}_{\mathrm{u}} \boldsymbol{u}=-\mathrm{K}_{\mathrm{u}}^{\dagger} \mathrm{K}_{\mathrm{g}} \boldsymbol{g}
$$

Here the symbol ${ }^{\dagger}$ denotes the adjoint. Further details, as well as an alternative variational motivation of the procedure, can be found in [11].

Note that so far we've been interested in approximate solutions of the homogeneous system (1), subject to boundary conditions that accommodate the prescribed incoming (guided) waves, together with arbitrary outgoing waves (cf. e.g. Refs. [27, 28] or the appendix of [11] for more formal statements). While these boundary conditions did not show up anywhere explicitly, they are built into the template (2) through the appropriate selection of contributing fields.

\subsection{Supermode analysis: eigenfrequencies of composite systems}

As a means to directly predict resonances of the composite systems, without carrying out frequency scans, we now look for - prospectively complex — values $\omega^{\mathrm{s}}$ where the system

$$
\boldsymbol{\nabla} \times \boldsymbol{H}-\mathrm{i} \omega^{\mathrm{s}} \epsilon_{0} \epsilon \boldsymbol{E}=0, \quad-\boldsymbol{\nabla} \times \boldsymbol{E}-\mathrm{i} \omega^{\mathrm{s}} \mu_{0} \boldsymbol{H}=0,
$$

subject to boundary conditions of outgoing waves only, permits nonzero solutions $\boldsymbol{E}, \boldsymbol{H}$. As before, approximate solutions are sought in the form of the template (2), now without any incoming waves (the respective 
coefficients $\boldsymbol{g}$ are zeroed). With the frequency parameter being replaced by the unknown value $\omega^{\mathrm{s}}$, one proceeds along the previous steps up to Eq. (8), of which only the upper left quadrant remains relevant:

$$
\mathrm{A}_{\mathrm{uu}} \boldsymbol{u}=\omega^{\mathrm{s}} \mathrm{B}_{\mathrm{uu}} \boldsymbol{u} .
$$

Eq. (12) constitutes a generalized eigenvalue problem. Solutions are pairs of eigenvectors $\boldsymbol{u}$ and eigenfrequencies $\omega^{\mathrm{s}}$. To distinguish the related fields from the original WGMs, we shall use the term "supermodes" for these resonances. One thus obtains a set of supermodes, each associated with a complex eigenfrequency $\omega^{\mathrm{s}}$, Q-factor $Q=\operatorname{Re} \omega^{\mathrm{s}} /\left(2 \operatorname{Im} \omega^{\mathrm{s}}\right)$, resonance wavelength $\lambda_{\mathrm{r}}=2 \pi \mathrm{c} / \operatorname{Re} \omega^{\mathrm{s}}$, linewidth $\Delta \lambda=\lambda_{\mathrm{r}} / Q$, and a mode profile, obtained by substituting the respective eigenvector into Eq. (2).

Depending on the specified field template the supermodes can include the power outlets provided by the bus waveguides; the Q-factors and linewithds then relate to the spectral properties of the waves that the composite open cavity sends out through the access channels.

Note that the terms "resonance" is being used in this paper in at least three different contexts: First there are the WGMs of the separate cavities (Section 2), given here as analytical solutions. Second, transmission resonances are observed as peaks and dips in the spectral scans of the scattering problems, determined by HCMT or an alternative method. Third, the supermodes of the composite systems are characterized by their complex eigenfrequencies, determined by HCMT-eigenvalue analysis with purely outgoing wave templates.

Figures 4, 8, and 9 show an excellent agreement between the resonance wavelengths and linewidths associated with the supermodes, and the peaks and dips in the spectral transmission curves. Note that, beyond observing that agreement, we do not establish any formal relation between the scans of the transmission problem, and the supermode analysis. A means to do that could be to employ variational procedures again, then with a template that combines the given incoming wave with an entire supermode, or a series of supermodes, of the composite system, in line with what has been carried out for 1-D problems in Refs. [14, 15, 16] (not done here). At least the field plots of e.g. Figure 5 provide some intuition on how this might work.

\subsection{Perturbations of whispering gallery resonances}

When applied to a template with only a single unknown, Eq. (12) permits one to derive an expression for the perturbation of the respective basis element by a small uniform change of permittivity. Assume that we investigate a cavity with permittivity function $\epsilon_{\mathrm{o}}$ which supports a resonance with field $\boldsymbol{E}_{\mathrm{o}}, \boldsymbol{H}_{\mathrm{o}}$ at frequency $\omega_{\mathrm{o}}$. This cavity is being perturbed slightly; the permittivity $\epsilon=\epsilon_{\mathrm{o}}+\Delta \epsilon$ characterizes the new configuration. We then look for the supermode of that configuration, using a template with merely the one given resonance. Eq. (12) can be evaluated explicitly for the supermode eigenfrequency. Neglecting terms $\sim \Delta \omega \Delta \epsilon$, the respective change in eigenfrequency $\Delta \omega$ can be given the form

$$
\Delta \omega=\frac{-\omega_{\mathrm{o}} \epsilon_{0} \iint \Delta \epsilon\left|\boldsymbol{E}_{\mathrm{o}}\right|^{2} \mathrm{~d} x \mathrm{~d} z}{\iint\left(\epsilon_{\mathrm{o}} \epsilon_{0}\left|\boldsymbol{E}_{\mathrm{o}}\right|^{2}+\mu_{0}\left|\boldsymbol{H}_{\mathrm{o}}\right|^{2}\right) \mathrm{d} x \mathrm{~d} z} .
$$

The approach requires that the original fields represent reasonable approximations of the actual fields of the perturbed structure. As exemplified by Figure 3 , this appears to be valid in case of small uniform perturbations [24], here a small change of the core refractive index of the cavity ring. However, one needs to be careful with shifts of dielectric interfaces, if discontinuous basis fields are involved [29, 30].

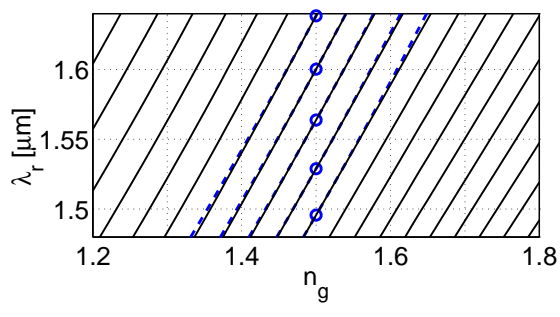

Figure 3: WGM resonance wavelengths $\lambda_{r}$ vs. core refractive index $n_{g}$, for rings with the parameters of Figure 1 (a) with $R=7.5 \mu \mathrm{m}$. The dashed lines indicate the slopes as predicted by Eq. (13), evaluated with the fields of the reference structure for $n_{g}=1.5$ (markers). 


\section{Examples}

Our C++-implementation of the HCMT model relies on the routine libraries [25, 23] for 2-D straight and bent slab waveguides. Commercial software for finite-difference-time-domain (FDTD, [31]) and frequency-domain finite-element (FD-FEM, [32]) simulations serves for benchmarking. The interpolation procedure as outlined in [4] has been applied for the fast evaluation of spectral scans in Figures 4, 8, and 9

Among the computational parameters, for the templates with radiating elements, the most notable influence must be expected from the extension of the computational window. For the present parameter set, however, this appears not to be critical. As an example, we could observe hardly any visible change of the transmission curves in a plot like Figure 4(a), if the window is enlarged from the original setting of $(20 \mu \mathrm{m})^{2}$ to $(45 \mu \mathrm{m})^{2}$. Accurate evaluation of the modal element overlaps (7) is required in all cases. The integrals are computed numerically by Gaussian quadrature [33], applied piecewise in case of non-smooth fields at dielectric interfaces, with stepsizes such that the overall results appear to be converged (checked at least occasionally).

\subsection{Single-ring or -disk filters}

Figure 4 summarizes the spectral properties of filter configurations with a single ring- or disk-cavity. Beyond the data from the present approach, the figure includes results from a series of alternative methods. The bend-mode based HCMT simulations view the cavity as a bent waveguide that supports eigenmodes with real frequency and complex angular wavenumber [4]. The "conventional" CMT model [34] splits the resonator into two coupler regions, each comprising adjacent segments of a bend and a straight waveguide. The guided wave interaction within these is modeled separately by standard codirectional coupled mode theory, with the coupler scattering matrices then embedded in an analytic resonator description. A quite satisfying nice overall agreement can be observed with the benchmark data from two numerical methods [32, 31].

As to be expected, slightly larger deviations appear for the resonances related to the first order WGMs of the micro-disk, where the larger radial extension of the WGM profiles leads to a stronger field overlap in the coupler regions (albeit not to a more efficient interaction). Obviously here the assumptions inherent in the HCMT template are less adequate.
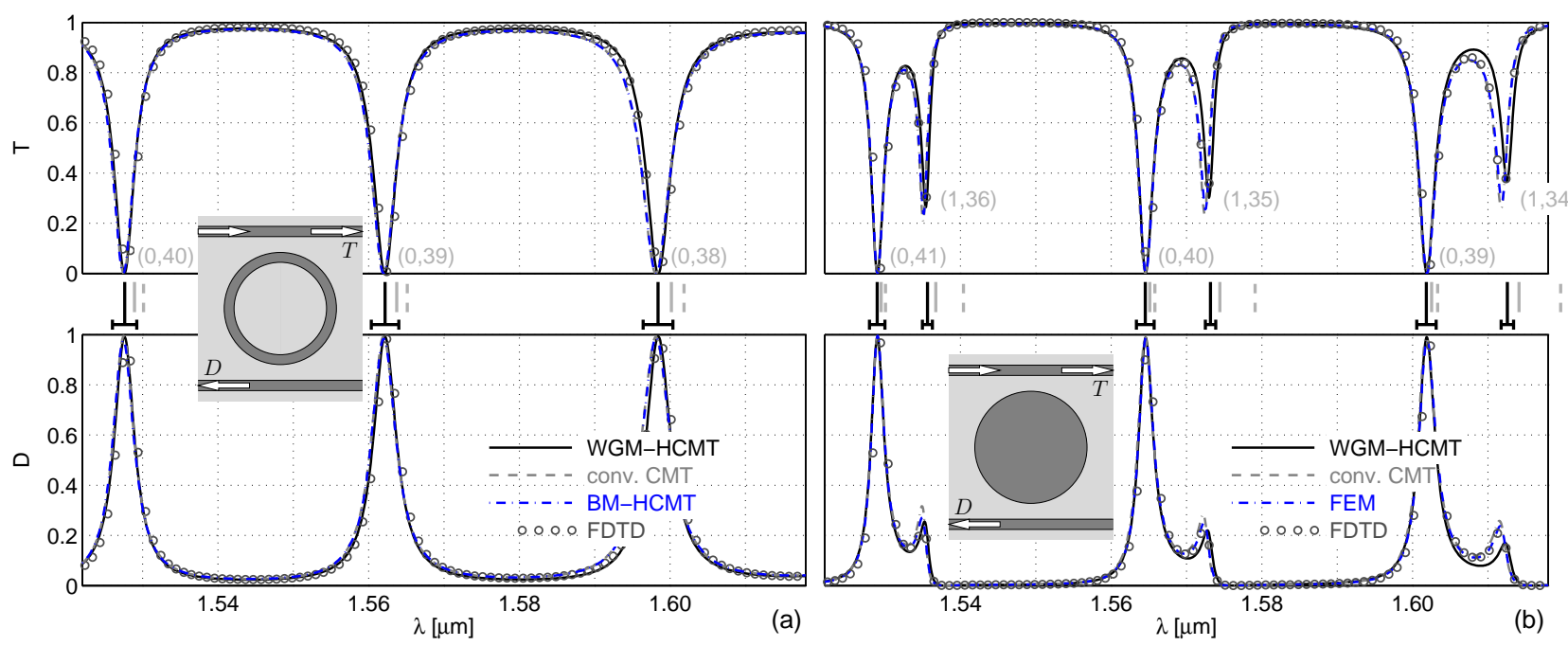

Figure 4: Transmission properties of micro-ring (a) and micro-disk resonators (b); directly transmitted power $T$ (top) and dropped optical power $D$ (bottom) as a function of the excitation wavelength $\lambda$. Parameters are as given for Figure 2 Results of different methods are compared, as explained further in the text: WGM-HCMT (continuous, the approach of this paper), conv-CMT (dashed, conventional CMT [34]), BM-HCMT (dash-dotted, (a) only, bend-mode-based HCMT [4]), FEM (dash-dotted, (b) only, finite element solver, commercial [32]), and FDTD (markers, finite-difference-timedomain, commercial [31]). Resonances are classified by the dominant contributing WGM. The marker lines in the central part are positioned at the respective resonance wavelengths: separate WGMs (light gray, continuous), WGMs perturbed by the bus waveguide permittivities (light gray, dashed), and HCMT supermodes (black, lower bars indicate the linewidths).

Figure 4 (a) shows WGM-HCMT results that are computed with a unidirectional template which includes WGMs $(0,37-41)$ of the ring. We also carried out WGM-HCMT scans with different field templates. If one 
uses $\operatorname{WGM}(0,39)$ only, then merely the respective dip/peak appears in the transmission/drop curves, positioned precisely (on the scale of Figure 4(a)) at the same spot, with an otherwise flat response. A bidirectional field template, with forward and backward waves in both bus channels and $\operatorname{WGMs}(0, \pm 37- \pm 41)$ leads to hardly any changes concerning the peak positions and widths, but to slightly lower power drop, i.e. to slightly higher losses. The levels of reflected power (upper left port in Figure 2) and forward-dropped guided power (lower right port) remain below $10^{-4}$ over the spectral range considered here.

When compared to the ring, the micro-disk supports WGMs of slightly higher quality, which leads to the narrower linewidths of the transmission resonances. These linewidths, however, are here obviously being dominated by the interaction between bus waveguides and WGMs: the first order WGMs, originally of lower quality, appear with narrower transmission resonances than the WGMs of fundamental radial order, that interact more effectively with the waves in the bus cores.

The central vertical lines in Figure 4 indicate the resonance wavelengths and linewidths associated with the (super-) modes of the device. While each individual WGM is clearly associated with one of the transmission extrema, there is still a noticeable difference between the resonance wavelengths of the separate WGMs and the positions of the transmission peaks. If one considers the bus channels as a mere permittivity perturbation, in line with the reasoning of Section 3.3 (minus sign in Eq. (13)), this leads to a further red-shift of the resonance positions; here the simple perturbational viewpoint is clearly inadequate. A supermode computation as outlined in Section 3.2, however, with a template that covers the WGMs of the cavity and the waves in the channel waveguides, permits one to predict the blue-shifted transmission resonances and their linewidths quite accurately. Table 1 collects some of the actual values that characterize these resonances; Figures 5 and 6 provide some impressions of the related field profiles.
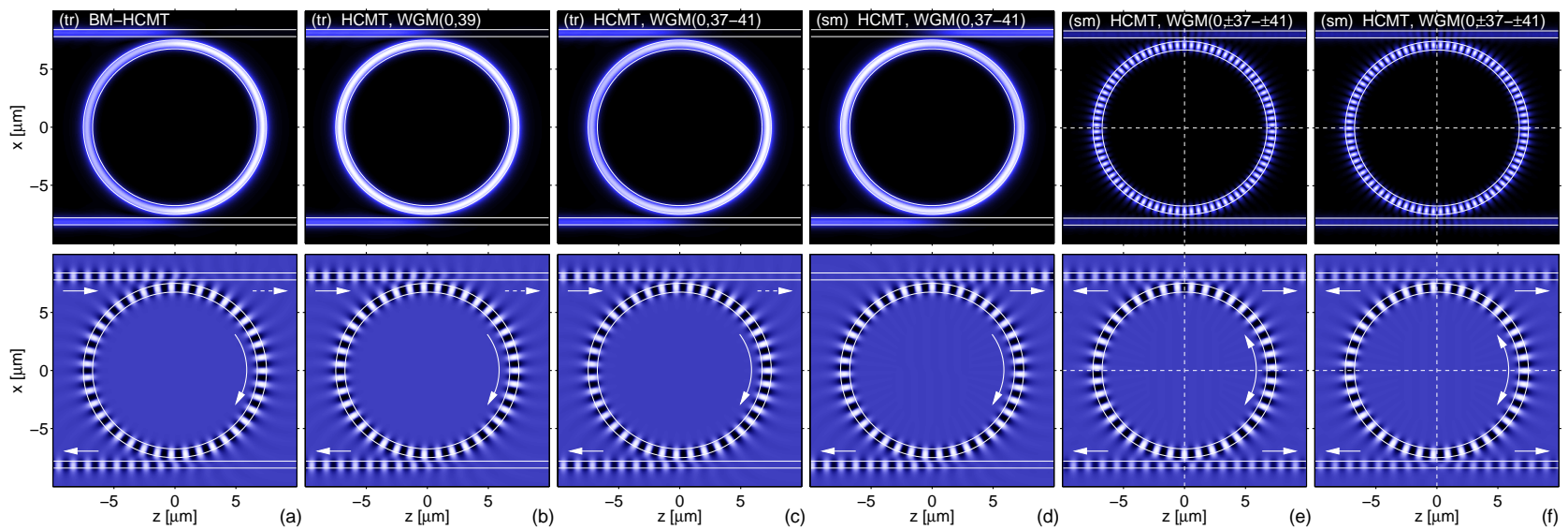

Figure 5: Field pattern related to the $\operatorname{WGM}(0,39)$ resonance of the ring filter in Figure 4(a). Panels (a-c) correspond to the transmission problem (tr), where a guided wave excitation is present in the upper left channel. Bend-mode-based HCMT results [4] (a) serve as reference. The WGM-HCMT simulations for (b) and (c) differ with respect to the HCMT template: Plots (b) are based on a template that includes unidirectional fields for both bus waveguides and merely the one $\operatorname{WGM}(0,39)$ for the cavity. Simulations (c) take also WGMs $(0,37-41)$ into account. Panels (d-f) show the results of supermode calculations (sm), based on either a unidirectional HCMT-template (d) or on a template where bidirectional variants of all fields are supplied (e, $f)$.

Panels $(\mathrm{a}-\mathrm{c})$ of Figure 5 relate to the transmission resonance of the ring for $\operatorname{WGM}(0,39)$. The bend-mode HCMT result (a), here the reference, shows noticeably different local intensities in the left and right half of the cavity. This could be attributed to waves that enter the cavity through the top coupler, propagate along half the ring, and leave the device through the bottom coupler, without contributing in the resonance effect. The single $\operatorname{WGM}(0,39)$ used in the template for panel (b) with its rotational symmetry can obviously not represent that feature. The template of (c) with a series of WGMs, however, leads to a field plot very similar to (a). One might thus just as well attribute the difference in local intensity to the interference of neighboring WGMs. In a plot of the coefficients $c_{j}$ from Eq. (2) versus excitation wavelength (not shown), one indeed observes a small nonzero amplitude for the respective WGMs of nearby angular order.

Supermode fields for the ring are illustrated in panels (d-f) of Figure 5, all with resonance wavelengths very close to (coinciding with) the $\operatorname{WGM}(0,39)$-transmission peak (cf. Table 1). Contrary to the unidirectional field (d) with its traveling waves in all cores (this also applies to $(\mathrm{a}-\mathrm{c})$ ), the time animation of $(\mathrm{e}, \mathrm{f})$ shows purely standing waves in the cavity, with outgoing waves in all four outlets. Applying the reasoning of [14, 15, 16] 


\begin{tabular}{|c|c|c|c|c|c|c|c|c|c|}
\hline Ring & $\lambda_{\mathrm{r}} / \mu \mathrm{m}$ & $Q$ & $\Delta \lambda / \mu \mathrm{m}$ & Fig. & Disk & $\lambda_{\mathrm{r}} / \mu \mathrm{m}$ & $Q$ & $\Delta \lambda / \mu \mathrm{m}$ & Fig. \\
\hline WGM $(0,39)$ & 1.56373 & $1.1 \cdot 10^{5}$ & $1.4 \cdot 10^{-5}$ & 1 (c) & WGM $(0,40)$ & 1.56514 & $8.2 \cdot 10^{5}$ & $1.9 \cdot 10^{-6}$ & 6 (a) \\
\hline pert. & 56516 & & & & pe & 1.56579 & & & \\
\hline M & $1.5621 x$ & & & & $(\mathrm{tr})$ & $1.5645 x$ & & & \\
\hline$(0,39)$ & $1.5622 x$ & & & $5(b)$ & (sr & 1.56454 & $6.7 \cdot 10^{2}$ & $2.3 \cdot 10^{-3}$ & \\
\hline & $1.5622 x$ & & & $5(c)$ & WGN & 1.57444 & $1.7 \cdot 10^{3}$ & $9.3 \cdot 10^{-4}$ & \\
\hline & 1.56219 & $4.3 \cdot 10^{2}$ & $3.7 \cdot 10^{-3}$ & $5(\mathrm{~d})$ & pe & 1.57904 & & & \\
\hline & 1.56223 & $4.5 \cdot 10^{2}$ & $3.5 \cdot 10^{-3}$ & $5(e)$ & (tr) & $1.5730 x$ & & & \\
\hline$(\mathrm{sm})_{\text {bd., o }}$ & 1.56215 & $4.0 \cdot 10^{2}$ & $3.9 \cdot 10^{-3}$ & 5 (f) & $(\mathrm{sm})_{\mathrm{ud} .}$ & 1.57320 & $1.1 \cdot 10^{3}$ & $1.4 \cdot 10^{-3}$ & f) \\
\hline
\end{tabular}

Table 1: Resonance wavelengths $\lambda_{\mathrm{r}}$, or peak locations, respectively, in case of the transmission problems (tr), quality factors $Q$ and spectral linewidths $\Delta \lambda$ associated with selected resonances of Figures 1, 4, 5, 6, Rows "pert" give the shifts of resonance wavelengths caused by the bus waveguide cores, if these are considered merely as permittivity perturbations. (tr) refers to extrema in the spectral transmission, computed by bend-mode HCMT (BM), or by the present WGM-HCMT with different templates. Rows (sm) contain the results of the HCMT-supermode analysis, with uni- (ud.) or bidirectional field templates (bd.).

one can imagine the field of the transmission resonance (c) emerging as the superposition of the unidirectional supermode (d) with a guided wave in the upper excitation channel, such that the fields in the upper right port interfere destructively, cancelling the direct transmission. The unidirectional supermode (d) in turn can be viewed as a superposition of the nearly degenerate bidirectional modes $(e, f)$, such that the waves cancel in the upper left and lower right outlets.
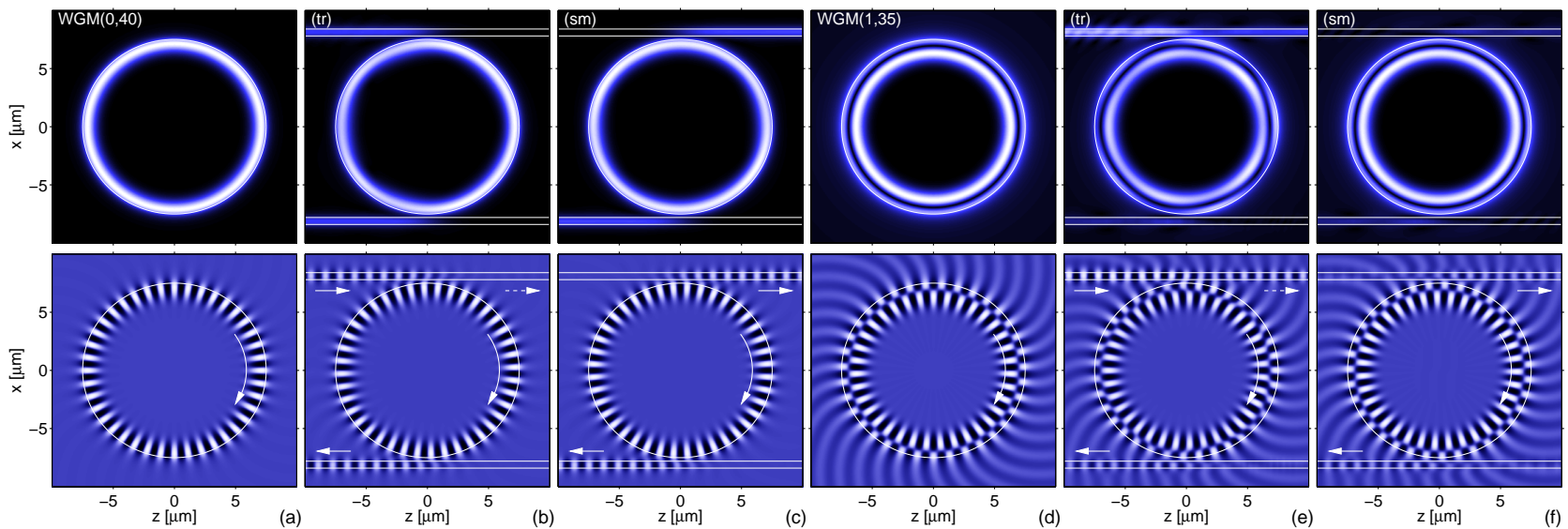

Figure 6: Field pattern related to central resonances of the disk filter in Figure 4(b). Panels $(b, c)$ correspond to the radial fundamental mode $\operatorname{WGM}(0,40)(a)$, panels $(e, f)$ show pattern for the first order $\operatorname{WGM}(1,35)(d)$. HCMT results for the resonant transmission (b), (e) are compared with the supermode fields of fundamental (c) and first order (f). All HCMT fields rely on a template that includes the WGMs $(0,38-42)$ and WGMs $(1,34-37)$.

Figure 6 collects a series of field examples for the micro-disk resonator. Features very similar to the ring are observed in panels $(\mathrm{a}-\mathrm{c})$ that concern the radially fundamental $\operatorname{WGM}(0,40)$. Fields $(\mathrm{d}-\mathrm{f})$ related to the $\operatorname{WGM}(1,35)$ of first radial order exhibit the radial nodal line and pronounced losses. As for the ring, for both sets one can imagine, at least approximately, the transmission resonance (tr) to be a superposition of the nearest supermodes (sm) and a guide wave in the upper channel, with almost complete (b) or partial (e) destructive interference in the upper right port. Contributions of both the supermodes (c) and (f) would explain the weak angular beating pattern [34] (most notable in (e)) of the transmission resonances.

With the recipes of Section 3.2 there are the means at hand to characterize directly the resonance properties of the composite filter devices, without carrying out frequency scans. The curves in Figure 7 shall serve as an example. The HCMT templates include the fields of the bus waveguides together with the WGMs $(0, \pm 37- \pm 41)$ of the cavity ring; results with uni- and bidirectional templates are compared. For the present parameter set, one observes hardly any difference between the uni- and bidirectional models, in line with the findings of Ref. [35]. The degeneracy of supermodes with opposite symmetry is being lifted slightly for pronounced field overlaps at nearly vanishing gaps (only just visible in part (a) for small $g$ ). In accordance with [17], depending on the bus core width (b), the cavity-bus-interaction causes a slightly red- or blue-shifted resonance of the composite system (both signs are possible). 


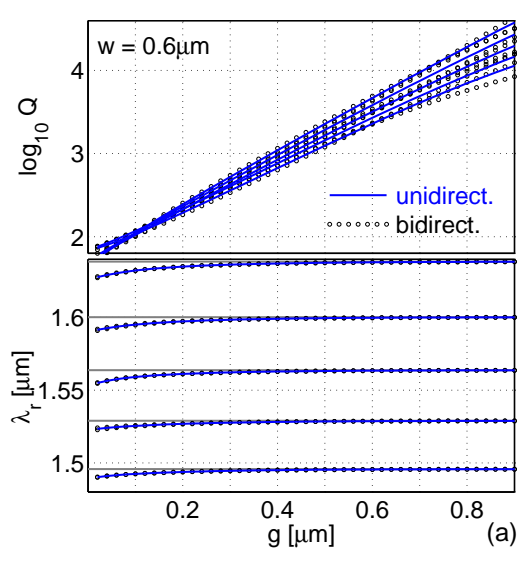

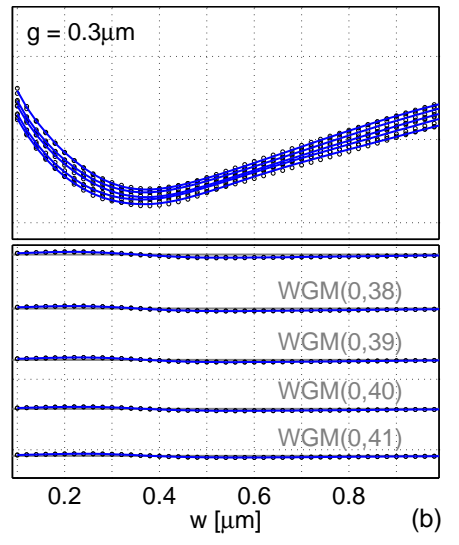

Figure 7: Resonance wavelengths $\lambda_{r}$ and $Q$ factors of ringresonators as in Figure 2, for varying gap $g$ (a), and bus width $w$ (b). The horizontal lines indicate the resonance wavelengths of the isolated cavity ring.

\subsection{Coupled resonator optical waveguide}

Linear series of coupled cavities received much attention in the recent past [36, 37], mainly for their optical delay properties, both theoretically and experimentally. Here we look at only one specific configuration adapted from [4], a series of nine of the former cavities. Figure 8 summarizes the results.

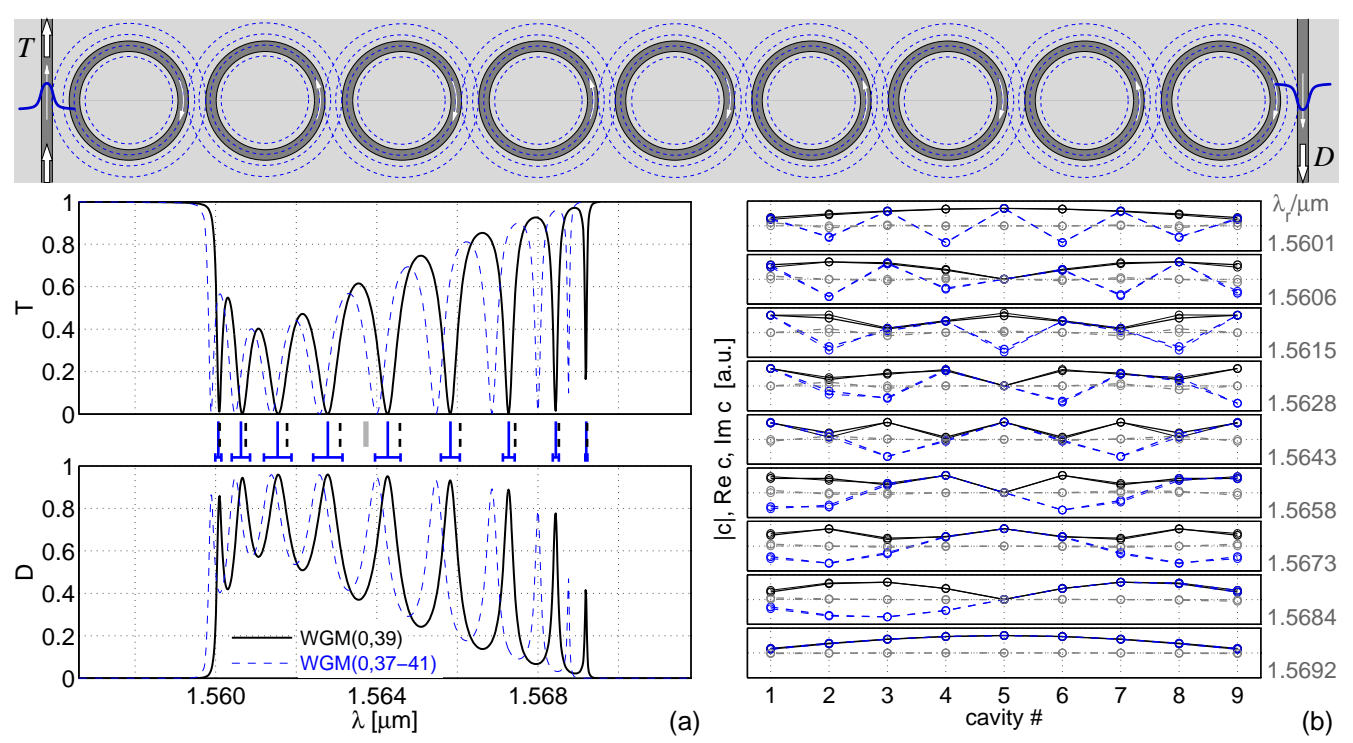

Figure 8: A coupled resonator optical waveguide (CROW), a series of cavities as introduced in Figure 2. (a): Spectrum of the device, relative transmission $T$ and power drop $D$ as as function of the excitation wavelength $\lambda$; unidirectional HCMT simulations based on templates with one WGM per cavity (bold lines) and with five WGMs per cavity (thin dashed curves). The central lines give the positions of the resonance wavelength of one separate ring (single bold line, gray), of the resonances for the series of rings without bus waveguides (dashed), and of the resonances of the complete system (continuous, here also the respective linewidths are shown), in all cases computed using templates with one WGM(0,39) per ring. (b): Supermode patterns for the system with bus waveguides (bold), and for the ring series only (thinner, mostly shadowed). Real parts (dashed), imaginary parts (dash-dotted), and absolute values (continuous) of the amplitudes attributed to the WGMs of neighboring cavities are connected to clarify the systematics.

We focus on the spectral region around the $\operatorname{WGM}(0,39)$-resonance of the individual rings. As for the filter of Section 4.1, one must expect that neighboring WGMs play a role. The transmission curves in Figure 8 (a), obtained with templates that include the $\operatorname{WGM}(0,39)$ and $\operatorname{WGMs}(0,37-41)$ for each ring, agree qualitatively, with only small shifts (on a scale of the free spectral range of the rings, $\approx 36 \mathrm{~nm}$, c.f. Figure $4(\mathrm{a})$ ) in the actual peak positions. We therefore restrict things to the model where each cavity is represented by a single WGM.

Supermode analysis for the chain of nine cavities, first without the bus channels, predicts that the original WGM splits into a series of nine supermodes, positioned close to, but not quite at, the transmission resonances. If the access waveguides are taken into account as well, the HCMT supermodes reflect accurately the peak positions and the linewiths of the transmission spectrum. Figure 8(b) compares the related "mode profiles", here the complex amplitudes assigned to the WGMs in the cavity series. One observes a systematic pattern of "harmonics" with a growing number of "nodes", where the fundamental resonance appears at the longest 
wavelength, i.e. at the lowest energy level. Only minor differences between the patterns for the systems without and with waveguides are visible.

Due to the semi-numerical nature of the HCMT approach there are no principal restrictions on distances, positioning, or (guided wave) excitation conditions. Small local or global changes in refractive index can be taken into account as perturbations, for supermode calculations or spectral scans. The present model should thus be a convenient means to to carry out ab-initio studies of further less-standard CROW-based circuits, like e.g. bends in CROW-based photonic molecules [38], defect-assisted CROWS [39], or tunable CROW based optical filters [40], always including the access waveguides.

\subsection{Three-ring photonic molecule}

As a last example we consider three identical rings, positioned at the corners of an equilateral triangle, and their excitation through a single access waveguide (Figure 9 (a)). Structures of this type have attracted interest for some time already. The parametric scattering-matrix model of Refs. [41, 42] predicts that the configuration can function as a resonant mirror / reflector. Without the bus channel, one might view the structure as a photonic "molecule", constituted by the three rings as photonic "atoms". A rigorous integral equation analysis of isolated molecules with emphasis on their Q-factors can be found in Ref. [43]. Further recent studies include the ab-initio HCMT model [4], based on the bend mode viewpoint, a parametric pathway analysis for purposes of application as a sensor [44], and an approximate analytical WGM-based description [45] that led to experimental observations [46]. Results of the present WGM-HCMT model are summarized in Figure 9.

(a)
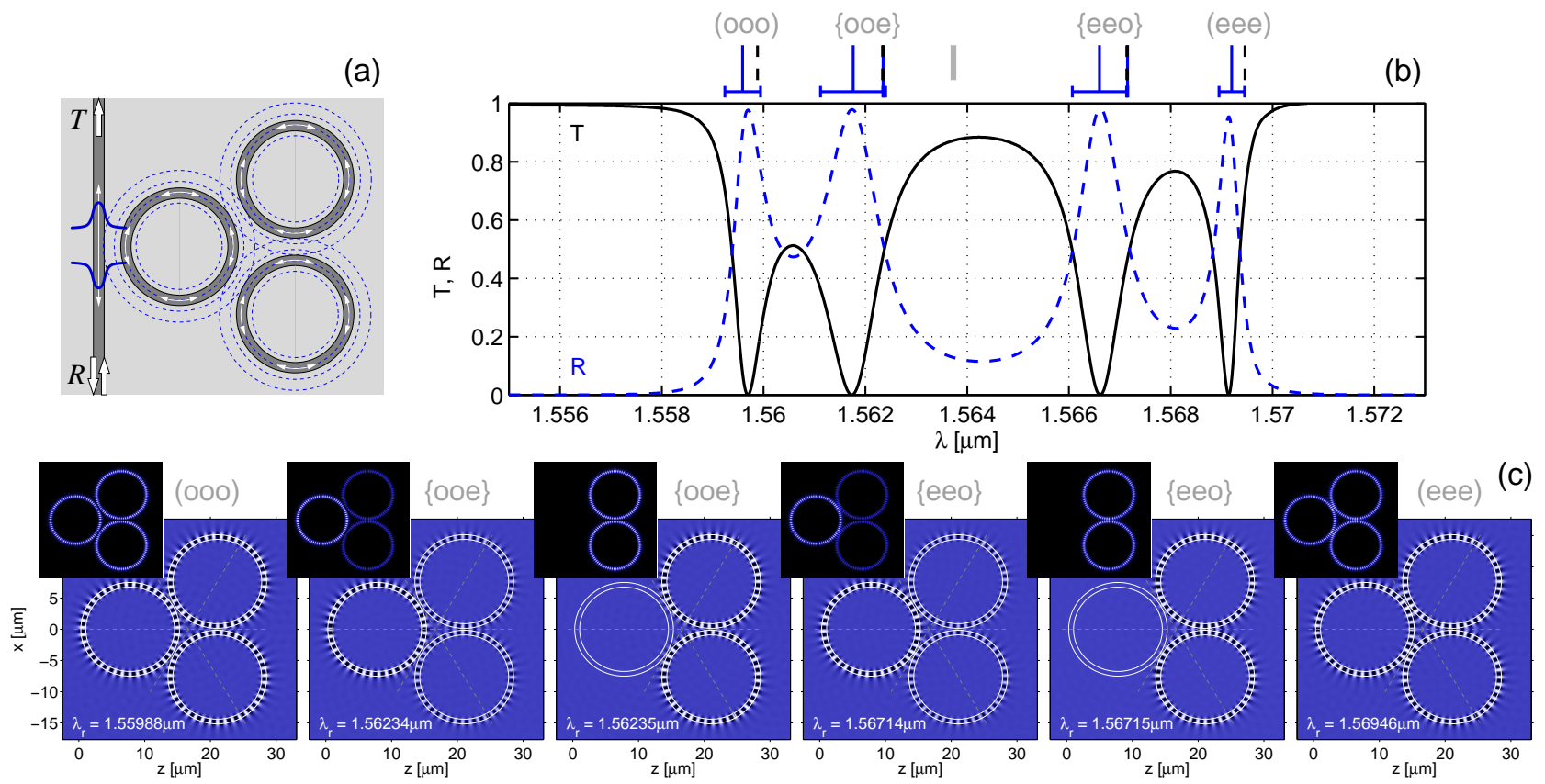

Figure 9: (a): Three-ring photonic molecule, excited by a straight bus waveguide. (b): Relative guided transmitted $(T)$ and reflected optical power $(R)$, as a function of excitation wavelength $\lambda$; bidirectional HCMT simulations with the $\operatorname{WGMs}(0, \pm 39)$ included for each ring. The vertical markers indicate the resonance $\operatorname{WGM}(0,39)$ of an individual ring (single bold gray line), the HCMT supermodes computed for the three-ring molecule only, without the bus channel (dashed), and the supermodes for the entire compound, consisting of molecule and waveguide (continuous, here also the respective linewidths are shown). (c): Supermode profiles predicted for the three-ring molecule, time-snapshots of the standing wave pattern (large panels) and absolute values (smaller insets) of the principal electric field component.

As before we look at the spectral region close to the $\operatorname{WGM}(0, \pm 39)$ resonance of the single rings. Bidirectional wave propagation is essential in this case; the cavities are thus represented in the HCMT template by six WGMs. Figure 9 b) shows the spectral guided wave transmission and reflection. As for the previous examples, one can only expect approximate results from the simple template. Comparison with the bend-mode HCMT in Ref. [4], however, shows that the spectral features are adequately captured by the WGM-HCMT model. The curves deviate slightly with respect to the precise peak positions, and with respect to the predicted maximum levels of reflection, for the three right-most reflection peaks. Note that these features cover a total wavelength range of about $10 \mathrm{~nm}$, roughly a quarter of the free spectral range of the individual rings. 
At the same time our present model can, at least to some degree, explain the formation of the resonances. Considering only the molecule without bus channels, the HCMT analysis predicts the six supermodes of Figure 9 (c). One observes purely standing waves in all cavities, with stationary nodal lines. This concerns a structure that is mirror-symmetric with respect to the three axes angled at $2 \pi / 3$, hinted at by the thin dashed lines in the field plots. One can thus expect modes with even (e) or odd parity (o) with respect to each of these axes. The panels in Figure 9 (c) and the related resonance wavelengths in (b) are classified accordingly. The "fundamental" supermode (eee) with the longest resonance wavelength / lowest energy exhibits the least "strained" profile, i.e. a field that is symmetric across all three lines. Likewise the supermode (ooo) with the most "strained" field appears with the shortest wavelength, or at highest energy, respectively. The four remaining supermodes come in two degenerate pairs. Of these, one is symmetric with respect to the horizontal axis, the other antisymmetrid 1 . Suitable superpositions of these two degenerate modes thus realize configurations where in turn each of the three cavities is "switched off", i.e. configurations with even or odd symmetry with respect to each of the three axes in turn, as exemplified by the third an fifth fields in the row.

Once a bus channel is placed as indicated in Figure 9(a), the incoming wave interacts only with those supermodes of the molecule that show non-negligible field strength in the vicinity of the bus core. Hence one observes merely four peaks, not six, in the transmission and reflection spectrum. The respective fields (not shown) resemble, in the region of the cavities, the first, second, fourth, and sixth profiles of Figure 9(c). With respect to the specific excitation through the waveguide, the supermodes in the third and fith panels behave as nonradiative "dark" states. In the present idealized model they are degenerate with the radiating "bright" states, the second and fourth profiles. Some small perturbation might thus lead to the excitation of sharp resonances of Fano-type [47], here in a comparably large dielectric integrated optic system.

As for the examples in Sections 4.1 and 4.2, the vertical lines in Figure 9 (b) show that the positions and linewidths of the peaks in the transmission spectrum can accurately be predicted by HCMT supermode analysis, if the bidirectional modes guided by the bus channel are taken into account. The respective supermodes are symmetric or antisymmetric with respect to the horizontal axis. Similar to the reasoning in Section 4.1 , the transmission resonances, here the states with vanishing reflection, can be thought of as a superposition of one of these supermodes with the upward traveling guided mode of the bus channel, such that the waves interfere destructively in the upper outlet.

\section{Concluding remarks}

The HCMT scheme can be used successfully with templates that involve known modes of open dielectric cavities. At least for the present configurations with high-Q cavities and thus moderately low losses, problems related to the outwards growing basis fields did not occur. Our ab-initio calculations are based on analytically computed WGMs of circular cavities. One obtains approximations for the full optical electromagnetic field. A series of examples show the versatility of the method. We've specifically looked at coupling-induced frequency shifts, which can conveniently be predicted by HCMT supermode analysis.

The scheme is inherently numerical in the sense that no analytical expressions for the amplitudes of the coupled modes emerge. Still, by extracting the respective numerical values, the coupled mode amplitudes can be made available for inspection and physical interpretation. Alternatively the method can be viewed as an approximate numerical (finite element) scheme, but one with very specific, structure-dependent nonlocal element functions. One operates at the intersection between "numerics" and "modeling"; convergence can be expected only up to what is built into the field template.

The extension to 3-D is still pending. While the formalism as given in this paper is directly applicable to 3D configurations, the present analytical WGMs would have to be replaced by numerical approximations, i.e. by basis fields computed through corresponding cavity eigenmode solvers [32]. For comparable circuits, the number of unknowns, i.e. the dimensions of the systems (10) and (12), would remain the same as in the 2-D case. However, apart from the bookkeeping, the efficient evaluation of the basis element overlaps (7) would constitute the major computational/numerical challenge.

\footnotetext{
${ }^{1}$ Due to the numerics (rectangular computational window, integration procedures) the horizontal axis is not strictly equivalent to the other two.
} 


\section{Acknowledgments}

The authors thank Hugo J.W.M. Hoekstra and Milan Maksimovic for many fruitful discussions.

\section{References}

[1] B. E. Little, S. T. Chu, W. Pan, and Y. Kokubun. Microring resonator arrays for VLSI photonics. IEEE Photonics Technology Letters, 12(3):323-325, 2000.

[2] F. Michelotti, A. Driessen, and M. Bertolotti, editors. Microresonators as building blocks for VLSI photonics, volume 709 of AIP conference proceedings. American Institute of Physics, Melville, New York, 2004.

[3] I. Chremmos, N. Uzunoglu, and O. Schwelb, editors. Photonic Microresonator Research and Applications. Springer Series in Optical Sciences, Vol. 156. Springer, London, 2010.

[4] M. Hammer. HCMT models of optical microring-resonator circuits. Journal of the Optical Society of America B, 27(11):2237-2246, 2010.

[5] A. Yariv. Universal relations for coupling of optical power between miroresonators and dielectric waveguide. Electronic Letters, 36(4):321-322, 2000.

[6] K. Okamoto. Fundamentals of Optical Waveguides. Academic Press, SanDiego, 2000.

[7] K. R. Hiremath, M. Hammer, R. Stoffer, L. Prkna, and J. Čtyroký. Analytical approach to dielectric optical bent slab waveguides. Optical and Quantum Electronics, 37(1-3):37-61, 2005.

[8] D. R. Rowland and J. D. Love. Evanescent wave coupling of whispering gallery modes of a dielectric cylinder. IEE Proceedings, Pt. J, 140(3):177-188, 1993.

[9] L. Prkna, J. Čtyroký, and M. Hubálek. Ring microresonator as a photonic structure with complex eigenfrequency. Optical and Quantum Electronics, 36(1/3):259-269, 2004.

[10] E. Franchimon. Modelling circular optical microresonators using whispering gallery modes. MSc thesis, University of Twente, Enschede, The Netherlands, 2010.

[11] M. Hammer. Hybrid analytical / numerical coupled-mode modeling of guided wave devices. Journal of Lightwave Technology, 25(9):2287-2298, 2007.

[12] P. T. Leung, S. Y. Liu, and K. Young. Completeness and orthogonality of quasinormal modes in leaky optical cavities. Physical Review A, 49(4):3057-3067, 1994.

[13] M. Bertolotti. Linear one dimensional resonant cavities. In F. Michelotti, A. Driessen, and M. Bertolotti, editors, Microresonators as building blocks for VLSI photonics, volume 709 of AIP conference proceedings, pages 19-47. American Institute of Physics, Melville, New York, 2004.

[14] M. Maksimovic, M. Hammer, and E. van Groesen. Field representation for optical defect microcavities in multilayer structures using quasi-normal modes. Optics Communications, 281(6):1401-1411, 2008.

[15] M. Maksimovic, M. Hammer, and E. van Groesen. Coupled optical defect microcavities in 1d photonic crystals and quasi-normal modes. Optical Engineering, 47(11):114601 1-12, 2008.

[16] M. Maksimovic. Optical resonances in multilayer structures. University of Twente, Enschede, The Netherlands, 2008. Ph.D. Thesis.

[17] M. A. Popović, C. Manolatou, and M. R. Watts. Coupling-induced resonance frequency shifts in coupled dielectric multi-cavity filters. Optics Express, 14(3):1208-1222, 2006.

[18] Q. Li, T. Wang, Y. Su, M. Yan, and M. Qiu. Coupled mode theory analysis of mode splitting in coupled cavity system. Optics Express, 18(8):8367-8382, 2010.

[19] O. Schwelb. On the nature of resonance splitting in coupled multiring optical resonators. Optics Communications, 281:1065-1071, 2008 .

[20] Q. Li, M. Soltani, A. H. Atabaki, S. Yegnanarayanan, and A. Adibi. Quantitative modeling of coupling-induced resonance frequency shift in microring resonators. Optics Express, 17(26):23474-23486, 2009.

[21] L. Y. M. Tobing, L. Tjahjana, S. Darmawan, and D. H. Zhang. Numerical and experimental studies of couplinginduced phase shift in resonator and interferometric integrated optics devices. Optics Express, 20(5):5789-5801, 2012.

[22] D. E. Amos. A portable package for Bessel functions of a complex argument and nonnegative order. http://www.netlib.org/amos/ 
[23] K. R. Hiremath. CIRCURS - Circular resonator simulator. http://www.zib.de/hiremath/circurs/

[24] C. Vassallo. Optical Waveguide Concepts. Elsevier, Amsterdam, 1991.

[25] M. Hammer. METRIC - Mode expansion tools for 2D rectangular integrated optical circuits. http://www.math.utwente.nl/ hammerm/Metric/

[26] J. D. Jackson. Classical Electrodynamics, 3rd. ed. Wiley, New York, 1998.

[27] B. Kettner. Detection of Spurious Modes in Resonance Mode Computations - Pole Condition Method. Freie Universität zu Berlin, Berlin, Germany, 2012. Dissertation.

[28] L. Zschiedrich. Transparent Boundary Conditions for Maxwells Equations: Numerical Concepts beyond the PML Method. Freie Universität zu Berlin, Berlin, Germany, 2009. Dissertation.

[29] M. Lohmeyer, N. Bahlmann, and P. Hertel. Geometry tolerance estimation for rectangular dielectric waveguide devices by means of perturbation theory. Optics Communications, 163(1-3):86-94, 1999.

[30] S. G. Johnson, M. Ibanescu, M. A. Skorobogatiy, O. Weisberg, J. D. Joannopoulos, and Y. Fink. Perturbation theory for Maxwell's equations with shifting material boundaries. Physical review E, 65:066611, 2002.

[31] PhoeniX BV, P.O. Box 545, 7500 AM Enschede, The Netherlands; http://www.phoenixbv.com/

[32] JCMwave GmbH, Haarer Str. 14a, 85640 Putzbrunn/Munich, Germany; http://www.jcmwave.com/.

[33] W. H. Press, S. A. Teukolsky, W. T. Vetterling, and B. P. Flannery. Numerical Recipes in C, 2nd ed. Cambridge University Press, 1992.

[34] K. R. Hiremath, R. Stoffer, and M. Hammer. Modeling of circular integrated optical microresonators by 2-D frequency-domain coupled mode theory. Optics Communications, 257(2):277-297, 2006.

[35] J. Čtyroký, I. Richter, and M. Šiňor. Dual resonance in a waveguide-coupled ring microresonator. Optical and Quantum Electronics, 38(9-11):781-797, 2006.

[36] A. Yariv, Y. Xu, R. K. Lee, and A. Scherer. Coupled-resonator optical waveguide: a proposal and analysis. Optics Letters, 24(11):711-713, 1999.

[37] A. Canciamilla, M. Torregiani, C. Ferrari, F. Morichetti, R. M. De La Rue, A. Samarelli, M. Sorel, and A. Melloni. Silicon coupled-ring resonator structures for slow light applications: potential, impairments and ultimate limits. Journal of Optics, 12:104008, 2010.

[38] S. V. Pishko, P. D. Sewell, T. M. Benson, and S. V. Boriskina. Efficient analysis and design of low-loss whisperinggallery-mode coupled resonator optical waveguide bends. Journal of Lightwave Technology, 25(9):2487-2494, 2007.

[39] O. Schwelb and I. Chremmos. Defect assisted coupled resonator optical waveguide: Weak perturbations. Optics Communications, 283(19):3686-3690, 2010.

[40] F. Morichetti, A. Melloni, A. Breda, A. Canciamilla, C. Ferrari, and M. Martinelli. A reconfigurable architecture for continuously variable optical slow-wave delay lines. Optics Express, 15(25):17273-17281, 2007.

[41] J. K. S. Poon, J. Scheuer, and A. Yariv. Wavelength-selective reflector based on a circular array of coupled microring resonators. IEEE Photonics Technology Letters, 16(5):1331-1333, 2004.

[42] O. Schwelb and I. Chremmos. Band-limited microresonator reflectors and mirror structures. In I. Chremmos, N. Uzunoglu, and O. Schwelb, editors, Photonic Microresonator Research and Applications, Springer Series in Optical Sciences, Vol. 156, pages 139-163. Springer, London, 2010.

[43] S. V. Boriskina. Theoretical prediction of a dramatic Q-factor enhancement and degeneracy removal of whispering gallery modes in symmetrical photonic molecules. Optics Letters, 31(3):338-340, 2006.

[44] S. I. Schmid, K. Xia, and J. Evers. Pathway interference in a loop array of three coupled microresonators. Physical Review A, 84:013808, 2011.

[45] C. Schmidt, A. Chipouline, T. Käsebier, E.-B. Kley, A. Tünnermann, and T. Pertsch. Observation of optical coupling in microdisk resonators. Physical Review A, 80:043841, 2009.

[46] C. Schmidt, M. Liebsch, A. Klein, N. Janunts, A. Chipouline, T. Käsebier, C. Etrich, F. Lederer, E.-B. Kley, A. Tünnermann, and T. Pertsch. Near-field mapping of optical eigenstates in coupled disk microresonators. Physical Review A, 85:033827, 2012.

[47] B. Gallinet and O. J. F. Martin. Ab initio theory of Fano resonances in plasmonic nanostructures and metamaterials. Physcal Review B, 83(23):235427, 2011. 